\title{
Dynamics of Liquid Lithium Atoms: Time Scales and Dynamic Correlation Functions
}

\author{
R.M. KhUSNUTdinOFF ${ }^{a, b, *}$ \\ ${ }^{a}$ Kazan (Volga region) Federal University, 420008 Kazan, Russia \\ ${ }^{b}$ Udmurt Federal Research Center of the Ural Branch of the Russian Academy of Sciences, 426068 Izhevsk, Russia
}

(Received May 23, 2018; revised version April 15, 2019; in final form December 12, 2019)

\begin{abstract}
Memory functions of the relaxation process of the density fluctuations of the lithium melt near the melting point on the basis of molecular dynamics data are calculated. It is established that the memory functions of the first four orders for the range of wave numbers corresponding to microscopic spatial scales are characterized by oscillating behavior. The frequency characteristics of the dynamic structure factor for a wide range of wave numbers are calculated. The convergence of the relaxation parameters $\Delta_{4}(k)$ and $\Delta_{5}(k)$ for the range of wave numbers from the hydrodynamic regime $(k \rightarrow 0)$ to the values of $k$ higher than the boundary of the first pseudo-Brillouin zone (for $k \approx 0.87 k_{m}$ ) are detected. The characteristic time scales of the process of structural relaxation of the density fluctuations are determined.
\end{abstract}

DOI: 10.12693/APhysPolA.137.267

PACS/topics: 61.20.-p, 61.05.F-, 67.25.du

\section{Introduction}

The memory function formalism introduced by Zwanzig and Mori $[1,2]$ is an invaluable tool to description of dynamic correlations in liquids from a theoretical point of view $[3,4]$. Dynamic correlations are described by the corresponding time correlation functions (TCF), which can be obtained from spectroscopic experiments (experiments on scattering of light and neutrons, inelastic X-ray scattering, etc.), and molecular dynamics simulations [5-7]. In the memory function formalism of Zwanzig-Mori, the time evolution of the TCF is associated with the so-called memory functions with the help of an infinite chain of integro-differential equations of the non-Markovian type. In describing various relaxation processes, it is essential to know the behavior of the memory function. A rich variety of model functions were proposed for this purpose, most of which have little physical justification. For example, Gaussian $[8,9]$ and exponential functions [10-12], delta function (the transition to the Markovian limit) [13], and hyperbolic secant [14-16], as well as their linear combinations $[17,18]$ were used to calculate velocity, transverse stress, energy current density correlation functions, and the corresponding transport coefficients for various types of liquids. Such memory functions often do not have any physical justification and the parameters entering into these expressions are found from the condition of the best agreement with the experiment. Determination of the behavior of the memory function is usually performed on the basis of the initial time correlation function by numerical solution of the integro-differential equation or

*corresponding author; e-mail: khrm@mail.ru the corresponding equation in the frequency mapping. Although the method is formally correct, the computed memory functions contain significant errors due to the numerical integration procedure and the instability of the numerical algorithm [19, 20]. Various approaches were proposed [21-23] to solve these problems. However, all methods are limited by the accuracy associated with the accuracy of the initial time correlation function, and the functions defined are limited only by the memory functions of lower orders. In the presented work, the considered approach is devoid of all these drawbacks. The lithium melt near the melting point was taken as the system under study, for which, as was shown earlier in [24], an adequate description of the structural and dynamic features is possible within the framework of molecular dynamics simulations with the pair interaction potential [25].

\section{Dynamic correlation functions and sum rules}

Let us consider a system consisting of $N$-atoms with mass $m$, in a volume $V$ and with a particle density $\rho=N / V$. The initial dynamic variable that allows to characterize the microscopic collective dynamics of the system is the local density fluctuation $\delta \rho(k, t)[26]$ :

$$
A_{0}(k, t)=\delta \rho(k, t)=\frac{1}{\sqrt{N}} \sum_{l}^{N} \mathrm{e}^{-\mathrm{i} \boldsymbol{k} \cdot \boldsymbol{r}_{l}(t)},
$$

where $\boldsymbol{r}_{l}(t)$ is the radius vector of the $l$-th particle at time $t$, and $\boldsymbol{k}$ is the wave vector. Using the GramSchmidt orthogonalization procedure [27]:

$$
\left\langle A_{n}(k)^{*}, A_{m}(k)\right\rangle=\delta_{n, m}\left\langle\left|A_{n}(k)\right|^{2}\right\rangle,
$$

one can get a set of orthogonal dynamic variables

$$
\boldsymbol{A}(k)=\left\{A_{0}(k), A_{1}(k), A_{2}(k), \ldots\right\} .
$$

The angle brackets denote the averaging over the canonical Gibbs ensemble and $\delta_{n, m}$ is the Kronecker symbol. 
To determine the time correlation function (TCF) of the density fluctuations we follow [28] and use

$$
F(k, t)=\frac{\left\langle A_{0}^{*}(k, 0) A_{0}(k, t)\right\rangle}{\left\langle\left|A_{0}(k, 0)\right|^{2}\right\rangle},
$$

which is related to the dynamic structure factor (experimentally measured value). This relation is

$$
S(k, \omega)=\frac{S(k)}{2 \pi} \operatorname{Re}\left(\int_{-\infty}^{\infty} F(k, t) \mathrm{e}^{\mathrm{i} \omega t} \mathrm{~d} t\right),
$$

and $S(k)$ is the static structure factor, or a zeroth frequency moment of the $S(k, \omega)$.

According to formalism of [29], the time evolution of the TCF of the density fluctuations can be represented with help of integro-differential equations of the form $\frac{\mathrm{d} M_{n}(k, t)}{\mathrm{d} t}=-\Delta_{n+1}(k) \int_{0}^{\infty} M_{n}(k, \tau) M_{n+1}(k, t-\tau) \mathrm{d} \tau$,

where

$$
M_{n}(k, t)=\frac{\left\langle A_{n}^{*}(k, 0) A_{n}(k, t)\right\rangle}{\left\langle\left|A_{n}(k, 0)\right|^{2}\right\rangle}
$$

is the so-called $n$-th order memory function, in particular, $M_{0}(k, t)=F(k, t)$. The quantities $\Delta_{n}(k)$ are the relaxation parameters. They have the dimension of the square of the frequency

$$
\omega^{(2 j)}(k)=\frac{\int \omega^{2 j} S(k, \omega) \mathrm{d} \omega}{\int S(k, \omega) \mathrm{d} \omega}, \quad j=1,2, \ldots
$$

and are related to the even frequency moments of the dynamic structure factor $S(k, \omega)[30]$ in the following way:

$$
\begin{aligned}
& \Delta_{1}(k)=\omega^{(2)}(k), \quad \Delta_{2}(k)=\frac{\omega^{(4)}(k)}{\omega^{(2)}(k)}-\omega^{(2)}(k), \quad \Delta_{3}(k)=\frac{\omega^{(6)}(k) \omega^{(2)}(k)-\left(\omega^{(4)}(k)\right)^{2}}{\omega^{(4)}(k) \omega^{(2)}(k)-\left(\omega^{(2)}(k)\right)^{3}}, \\
& \Delta_{4}(k)=\frac{1}{\Delta_{1}(k) \Delta_{2}(k) \Delta_{3}(k)}\left\{\omega^{(8)}(k)-\Delta_{1}(k)\left[\left(\Delta_{1}(k)+\Delta_{2}(k)\right)^{3}+\Delta_{2}(k)\left(\Delta_{3}(k)\right)^{2}+2 \Delta_{2}(k) \Delta_{3}(k)\left(\Delta_{1}(k)+\Delta_{2}(k)\right)\right]\right\}, \\
& \Delta_{5}(k)=\frac{1}{\Delta_{1}(k) \Delta_{2}(k) \Delta_{3}(k) \Delta_{4}(k)}\left\{\omega^{(10)}(k)-2 \omega^{(8)}(k)\left[\Delta_{1}(k)+\Delta_{2}(k)+\Delta_{3}(k)+\Delta_{4}(k)\right]\right. \\
& +\omega^{(6)}(k)\left[\left(\Delta_{1}(k)\right)^{2}+\left(\Delta_{2}(k)\right)^{2}+\left(\Delta_{3}(k)\right)^{2}+2 \Delta_{2}(k)\left(\Delta_{1}(k)+\Delta_{3}(k)\right)+4 \Delta_{1}(k) \Delta_{3}(k)\right] \\
& \left.-\omega^{(4)}(k)\left[2 \Delta_{1}^{2}(k)\left(\Delta_{3}(k)+\Delta_{4}(k)\right)+2 \Delta_{1}(k)\left(\Delta_{2}(k)+\Delta_{3}(k)\right)\left(\Delta_{3}(k)+2 \Delta_{4}(k)\right)+2 \Delta_{2}(k) \Delta_{4}(k)\left(\Delta_{2}(k)+\Delta_{3}(k)\right)\right]\right\} \\
& +\Delta_{4}(k)+2 \Delta_{1}(k)+2 \Delta_{1}(k) \frac{\Delta_{1}(k)}{\Delta_{2}(k)}+\frac{\left(\Delta_{1}(k)\right)^{2} \Delta_{3}(k)}{\Delta_{2}(k) \Delta_{4}(k)}
\end{aligned}
$$

Note that for the classical system only the even frequency moments are non-zero. Therefore, the second, the fourth, and the sixth frequency moments of the dynamic structure factor $S(k, \omega)$ in the case of a spherical pair potential $\mathcal{U}(r)$ can be found using the microscopic expressions [31, 32]:

$$
\begin{aligned}
\omega^{(2)}(k) & =\frac{k_{\mathrm{B}} T}{m} \frac{k^{2}}{S(k)}, \quad \omega^{(4)}(k)=\frac{3}{S(k)}\left(\frac{k_{\mathrm{B}} T}{m} k^{2}\right)^{2}+\frac{\rho k_{\mathrm{B}} T}{m^{2} S(k)} k^{2} \int \mathrm{d} \boldsymbol{r} g(r)[1-\cos (\boldsymbol{k} \cdot \boldsymbol{r})] \nabla_{l}^{2} \mathcal{U}(r) \\
\omega^{(6)}(k) & =\frac{15}{S(k)}\left(\frac{k_{\mathrm{B}} T}{m} k^{2}\right)^{3}+\frac{15 \rho}{m S(k)}\left(\frac{k_{\mathrm{B}} T}{m} k^{2}\right)^{2} \int \mathrm{d} \boldsymbol{r} g(r) \nabla_{l}^{2} \mathcal{U}(r)+\frac{6 \rho k_{\mathrm{B}}^{2} T^{2}}{m^{3} S(k)} k^{3} \int \mathrm{d} \boldsymbol{r} g(r) \nabla_{l}^{3} \mathcal{U}(r) \sin (\boldsymbol{k} \cdot \boldsymbol{r}) \\
+ & \frac{2 \rho^{2} k_{\mathrm{B}} T}{m^{3} S(k)} k^{2} \int \mathrm{d} \boldsymbol{r} g(r)\left(\nabla \nabla_{l} \mathcal{U}(r)\right)^{2}[1-\cos (\boldsymbol{k} \cdot \boldsymbol{r})] \\
+ & \frac{\rho^{2} k_{\mathrm{B}} T}{m^{3} S(k)} k^{2} \iint \mathrm{d} \boldsymbol{r} \mathrm{d} \boldsymbol{r}^{\prime} g_{3}\left(\boldsymbol{r}, \boldsymbol{r}^{\prime}\right)\left(\nabla \nabla_{l} \mathcal{U}(r)\right)\left(\nabla^{\prime} \nabla_{l}^{\prime} \mathcal{U}\left(r^{\prime}\right)\right)\left[1-\cos (\boldsymbol{k} \cdot \boldsymbol{r})-\cos \left(\boldsymbol{k} \cdot \boldsymbol{r}^{\prime}\right)+\cos \left(\boldsymbol{k} \cdot\left(\boldsymbol{r}-\boldsymbol{r}^{\prime}\right)\right)\right]
\end{aligned}
$$


Here, the subscript $l$ denotes the longitudinal component parallel to the vector $\boldsymbol{k}, g(r)$ is the radial distribution function of two particles, $g_{3}\left(\boldsymbol{r}, \boldsymbol{r}^{\prime}\right)$ is a threeparticle radial distribution function, $k_{\mathrm{B}}$ is the Boltzmann constant, and $m$ is the particle mass. The expressions for higher-order frequency moments have a rather complex form and contain many-particle radial distribution functions, the finding of which presents a separate and complex problem [33]. Consequently, the correct definition of the frequency moments above the fourth order based on microscopic expressions is not always possible. In turn, the values of the frequency moments $\omega^{(2 m)}(k)$ ( $m=1,2,3, \ldots)$ obtained on the basis of the integration of the spectral density $S(k, \omega)$ with the help of $(8)$, are characterized by significant errors, which almost always lead to unphysical results.

Relaxation parameters can also be determined numerically based on molecular dynamics simulation data in accordance with their basic definitions [30]:

$$
\Delta_{n}(k)=\frac{\left\langle\left|A_{n}(k, 0)\right|^{2}\right\rangle}{\left\langle\left|A_{n-1}(k, 0)\right|^{2}\right\rangle}, \quad n=1,2 \ldots
$$

The dynamic variables are determined by the relations

$$
\begin{aligned}
& A_{1}(k, t)=\frac{\partial A_{0}(k, t)}{\partial t}=-\frac{\mathrm{i}}{\sqrt{N}} \sum_{l}^{N}\left(\boldsymbol{k} \cdot \boldsymbol{v}_{l}(t)\right) \mathrm{e}^{-\mathrm{i} \boldsymbol{k} \cdot \boldsymbol{r}_{l}(t)}, \\
& A_{2}(k, t)=\frac{\partial A_{1}(k, t)}{\partial t}+\Delta_{1} A_{0}(k, t)= \\
& \quad \frac{1}{\sqrt{N}} \sum_{l}^{N}\left\{\Delta_{1}-\left(\boldsymbol{k} \cdot \boldsymbol{v}_{l}(t)\right)^{2}-\mathrm{i}\left(\boldsymbol{k} \cdot \boldsymbol{a}_{l}(t)\right)\right\} \mathrm{e}^{-\mathrm{i} \boldsymbol{k} \cdot \boldsymbol{r}_{l}(t)}, \\
& \ldots \quad \\
& A_{n}(k, t)=\frac{\partial A_{n-1}(k, t)}{\partial t}+\Delta_{n-1} A_{n-2}(k, t) .
\end{aligned}
$$

Thus, it is possible to perform a fairly correct estimate for the memory functions and the frequency characteristics of the dynamic structure factor $S(k, \omega)$, on the basis of molecular dynamics simulation data.

\section{Simulation details}

Molecular dynamics simulation of the lithium melt in the NVT-ensemble at the temperature $T=475 \mathrm{~K}$ with the number density $\rho=0.0445^{-3}$ was performed. The system consisted of $N=4394$ atoms enclosed in a cubic cell with periodic boundary conditions. Interaction between atoms was realized with the help of a pair potential [25]. The equations of motion were integrated using the velocity Verlet algorithm with time step $\tau=0.01 \mathrm{ps}$ [35]. To bring the system to thermodynamic equilibrium and to calculate the spectral characteristics, 100000 and 2000000 time steps were made, respectively.

In Fig. 1, we have compared $g(r)$ and $S(k)$ for liquid lithium at temperature $T=475 \mathrm{~K}$ with the experimental X-ray diffraction data [34]. These functions $g(r)$
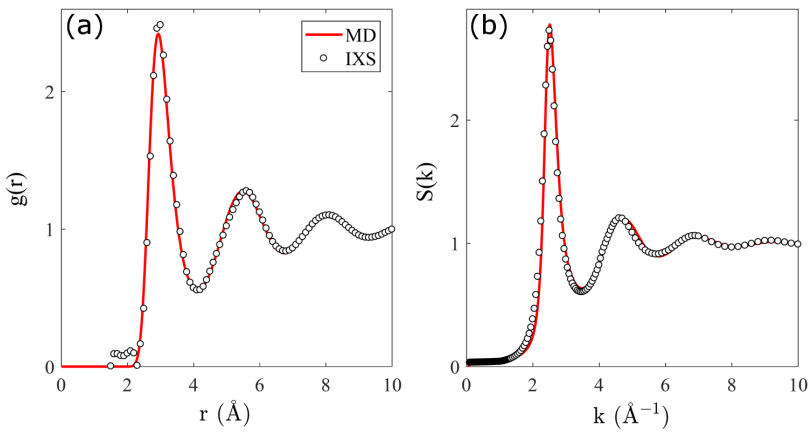

Fig. 1. (a) Radial atomic distribution function for liquid lithium at temperature $T=475 \mathrm{~K}$. The solid lines are the results of simulation of the atomic dynamics with the effective pseudopotential [25]. ( $\circ \circ \circ$ ) - experimental data on X-ray diffraction [34]. (b) Static structure factor of the lithium melt.

and $S(k)$ are calculated using the effective pseudopotential [25]. The simulation results are seen to reliably characterize the fine structure of the system under study and to exactly reproduce the experimental data.

\section{Simulation results and numerical calculations}

The dynamic structure factor $S(k, \omega)$ of the system under study is connected with the intensity of inelastic X-ray scattering $I(k, \omega)$ by the relation [36]

$$
\begin{gathered}
I(k, \omega)=E(k) \int \frac{\hbar \omega^{\prime} / k_{\mathrm{B}} T}{1-\mathrm{e}^{-\hbar \omega^{\prime} / k_{\mathrm{B}} T}} \\
\quad \times R\left(k, \omega-\omega^{\prime}\right) S\left(k, \omega^{\prime}\right) \mathrm{d} \omega^{\prime},
\end{gathered}
$$

where $E(k)$ denotes the normalized form factor, $R(k, \omega)$ is the experimental resolution function.

In Fig. 2, the molecular dynamics results of $I(k, \omega)$ are compared with the experimental IXS data [37]. As can be seen, the numerical results for the dynamic structure factor has excellent agreement with experimental data.
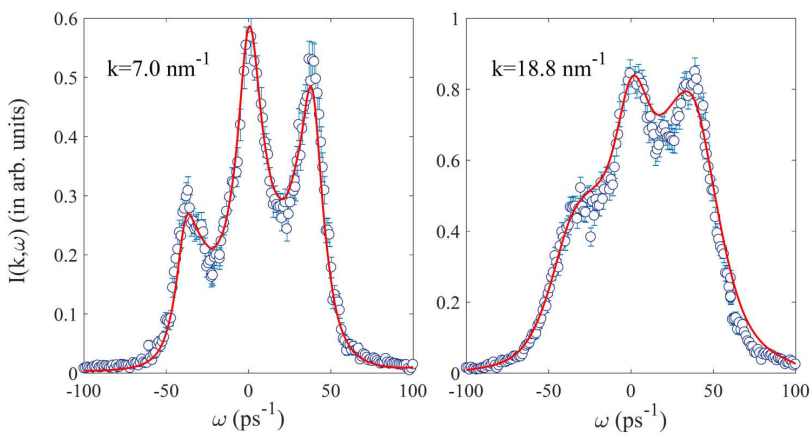

Fig. 2. The intensity of inelastic X-ray scattering $I(k, \omega)$ in liquid lithium at the temperature $T=475$ $\mathrm{K}$. The solid lines present the molecular dynamics results with the pair-potential [25] convoluted with the experimental resolution and involving the detailed balance condition. ( $\left(\begin{array}{lll}\circ & \circ & \circ\end{array}\right)$ - the experimental data [37]. 


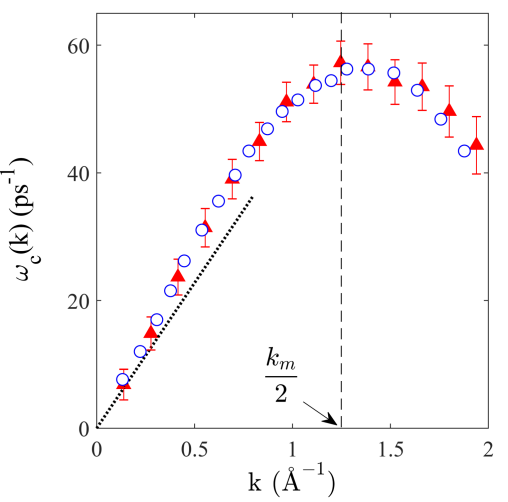

Fig. 3. Dispersion of collective excitations of the longitudinal polarization for liquid lithium at the temperature $T=475 \mathrm{~K}$. ( $\mathbf{\Delta} \boldsymbol{\Delta} \boldsymbol{\Delta})$ - the results of molecular dynamics simulation. ( $\circ \circ \circ)$ - the experimental data on inelastic scattering of X-rays [37]. Dotted line represents the extrapolated hydrodynamic result $\omega_{c}(k)=c_{s} k$, where $c_{s}=4554 \mathrm{~m} / \mathrm{s}$ is the adiabatic sound velocity. The vertical dashed straight line corresponds to the boundary of the first pseudo-Brillouin zone.

Figure 3 shows the dispersions of the high-frequency $I(k, \omega)$ peak determined from the molecular dynamics simulation and experimental data on inelastic X-rays scattering [37]. The results of molecular dynamics simulation are seen to precisely reproduce the experimental data. Moreover, all (experimental and simulation) results indicate the presence of the so-called positive dispersion effect [5] in the microscopic region under study: the values of $\omega_{c}(k)$ exceed the values predicted by the usual hydrodynamic theory with a linear dispersion [29], and a sound velocity $c_{s}=4554 \mathrm{~m} / \mathrm{s}$.

Figure 4 presents the calculated memory functions for liquid lithium at $T=475 \mathrm{~K}$ obtained with help of the relations (7) and (12) based on the data of molecular dynamics simulation. In Fig. 4, all memory functions for the considered values of wave numbers are characterized by oscillating behavior.

Figure 5a represents the relaxation parameters of the dynamic structure factor for lithium melt, calculated using the relations (11) and (12) based on the molecular dynamics simulation data. In Fig. 5a, all parameters with an increase in the wave number have a similar $k$-dependence. The behavior of the parameters $\Delta_{4}(k)$ and $\Delta_{5}(k)$ has a single character from the hydrodynamic regime to the range of the wave number $k \approx 2.2 \AA^{-1}$, which corresponds to value $k \approx 0.87 k_{m}$ ( $k_{m}$ is the position of the main maximum in the static structure factor $S(k))$. However, the parameters $\Delta_{4}(k)$ and $\Delta_{5}(k)$ diverge with increasing values of wave numbers. The inset in Fig. 5 represents the ratios of the frequency relaxation parameters $\Delta_{n}(k) / \Delta_{1}(k)$ for $n=2,3,4,5$ and for the range of values of high wave numbers. As can be seen, the results of numerical calculations for the values of $\Delta_{n}(k) / \Delta_{1}(k)$ correctly reproduce the short-wave asymptotic (the region of the free-particle dynamics).

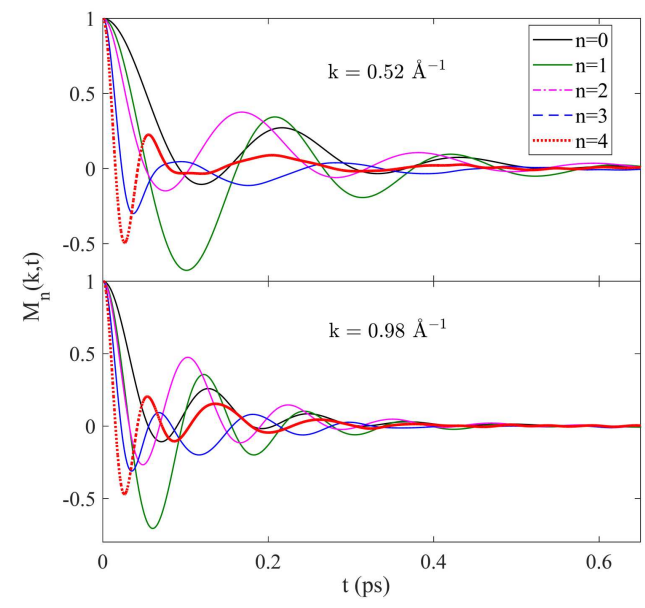

Fig. 4. Time correlation function of the density fluctuations $F(k, t)=M_{0}(k, t)$ and the memory function $M_{n}(k, t)$ of the first four orders $(n=1,2,3,4)$ of the lithium melt at the temperature $T=475 \mathrm{~K}$ for the wave numbers $k=0.52 \AA^{-1}$ and $k=0.98 \AA^{-1}$.
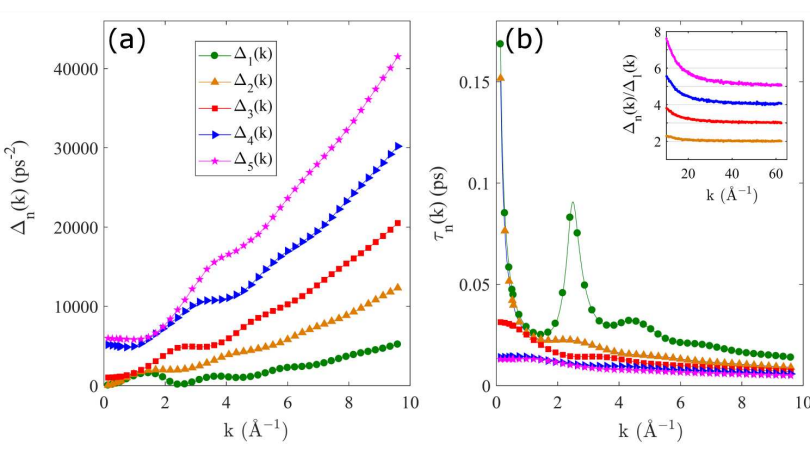

Fig. 5. (a) Relaxation parameters of the dynamic structure factor of lithium melt at the temperature $T=475 \mathrm{~K}$, obtained on the basis of molecular dynamics simulation data with the pair interatomic interaction potential [25]. (b) Time scales characterizing the relaxation processes of the density fluctuations in liquid lithium near the melting point. Inset: ratio of the frequency relaxation parameters $\Delta_{n}(k) / \Delta_{1}(k)$ for $n=2,3,4$ and 5 .

Figure 5b presents the time scales $\tau_{n}(k)=1 / \sqrt{\Delta_{n}(k)}$ characterizing the relaxation processes of the density fluctuations in liquid lithium near the melting point. In Fig. 5b, the timescales of the relaxation processes related to the dynamical variables $A(n)(n \geq 4)$ are approximately equal, i.e., $\tau_{n}(k) \approx \tau_{n+1}(k)$ [38]. Consequently, the dynamics of the density fluctuations in liquid lithium is characterized by some minimal time scale, which is $\tau_{\text {min }} \sim 10^{-15} \div 10^{-14} \mathrm{~s}$.

\section{Conclusion and discussions}

The study of the nature of the collective modes in liquid metals belongs to one of the most fundamental problems of condensed matter physics. So, for example, liquid 
metals are suitable candidates to test the proposed theories (mode-coupling theories, theory of the generalized collective modes, classic theory of moments, etc.) of the microscopical collective dynamics in a liquid [29]. Within these approaches, memory functions and relaxation parameters play a key role. Therefore, all the relaxation mechanisms controlling the collective and single-particle dynamics, are introduced at the level of the corresponding second or higher order memory functions.

In this paper we have presented molecular dynamic simulation results of the structural relaxation of the density fluctuations of the lithium melt near the melting point. The frequency characteristics of the dynamic structure factor for a wide range of wave numbers are obtained. It is shown that the ratios of the relaxation parameters $\Delta_{n}(k) / \Delta_{1}(k)$ for $n=2,3,4,5$ in the short-wave region satisfy for the condition: $\lim _{k \rightarrow \infty} \Delta_{n}(k) / \Delta_{1}(k)=n$. The behavior of the relaxation parameters $\Delta_{4}(k)$ and $\Delta_{5}(k)$ has a unified character for the range of values of wave numbers from the hydrodynamic regime $(k \rightarrow 0)$ to the values of $k$ above the boundary of the first pseudo-Brillouin zone $\left(k>k_{m} / 2\right)$. The characteristic time scales of the process of structural relaxation of the density fluctuations are determined. The memory functions up to the fourth order for the time correlation function of the density fluctuations for liquid lithium at the temperature $T=475 \mathrm{~K}$ were calculated.

\section{Acknowledgments}

The authors are grateful to Prof. L.E. González (Universidad de Valladolid, Valladolid, Spain), who provided information (parameters and characteristics) on the liquid lithium pseudopotential. This work was supported by the Russian Foundation for Basic Research (project No. 18-02-00407-a).

\section{References}

[1] R. Zwanzig, Phys. Rev. 124, 1338 (1961).

[2] H. Mori, Prog. Theor. Phys. 33, 423 (1965).

[3] J.P. Boon, S. Yip, Molecular Hydrodynamics, McGraw-Hill, New York 1980.

[4] R.M. Khusnutdinov, A.V. Mokshin, I.I. Khadeev, J. Surf. Invest. X-ray Synchrotr. Neutron Tech. 8, 84 (2014).

[5] T. Scopigno, G. Ruocco, F. Sette, Rev. Mod. Phys. 77, $881(2005)$.

[6] Yu.D. Fomin, V.N. Ryzhov, E.N. Tsiok, V.V. Brazhkin, J. Phys. Condens. Matter 29, 345401 (2017).

[7] R.M. Khusnutdinoff, Coll. J. 78, 225 (2016).

[8] J.P. Mithen, J. Daligault, B.J.B. Crowley, G. Gregori, Phys. Rev. E 84, 046401 (2011).

[9] J.P. Mithen, Phys. Rev. E 89, 013101 (2014).

[10] B.J. Berne, J.P. Boon, S.A. Rice,J. Chem. Phys. 45, 1086 (1966).

[11] U. Bafile, E. Guarini, F. Barocchi, Phys. Rev. E 73, 061203 (2006).
[12] F. Aliotta, J. Gapinski, M. Pochylski, R.C. Ponterio, F. Saija, C. Vasi, Phys. Rev. E 84, 051202 (2011).

[13] A. Rahman, Phys. Rev. 136, A405 (1964).

[14] K. Tankeshwar, K.N. Pathak, S. Ranganathan, J. Phys. C Solid State Phys. 20, 5749 (1987).

[15] K. Tankeshwar, K.N. Pathak, S. Ranganathan, J. Phys. C Solid State Phys. 21, 3607 (1988).

[16] S. Singh, K. Tankeshwar, Phys. Rev. E 67, 012201 (2003).

[17] A.V. Mokshin, R.M. Yulmetev, R.M. Khusnutdinoff, P. Hänggi, J. Exp. Theor. Phys. 103, 841 (2006).

[18] M.J. Nuevo, J.J. Morales, D.M. Heyes, Phys. Rev. E 55, 4217 (1997).

[19] P.A. Egelstaff, Phys. Chem. Liq. 16, 293 (1987).

[20] R. Vogelsang, C. Hoheisel, J. Stat. Phys. 54, 315 (1989).

[21] M.I. Barker, T. Gaskell, J. Phys. C 5, 353 (1972).

[22] L. Sjogren, J. Phys. C 13, 705 (1980).

[23] G.R. Kneller, K. Hinsen, J. Chem. Phys. 115, 11097 (2001).

[24] R.M. Khusntdinoff, B.N. Galimzyanov, A.V. Mokshin, J. Exp. Theor. Phys. 126, 83 (2018).

[25] L.E. González, D.J. González, M. Silbert, J.A. Alonso, J. Phys. Condens. Matter 5, 4283 (1993).

[26] R.M. Khusnutdinoff, Acta Phys. Pol. A 126, 293 (2016).

[27] M.C. Reed, B. Simon, Methods of Modern Mathematical Physics, Academic, New York 1972, Mir, Moscow 1977.

[28] U. Balucani, M. Zoppi, Dynamics of the Liquid State, Clarendon, Oxford 1994.

[29] J.P. Hansen, I.R. McDonald, Theory of Simple Liquids, Academic Press, New York 2006.

[30] A.V. Mokshin, R.M. Yulmetyev, R.M. Khusnutdinoff, P. Hänggi, J. Phys. Condens. Matter 19, 046209(1) (2007).

[31] E. Michler, H. Hann, P. Schofield, J. Phys. F Met. Phys. 7, 869 (1977).

[32] R.M. Khusnutdinoff, A.V. Mokshin, S.G. Menshikova, A.L. Beltyukov, V.I. Ladyanov, J. Exp. Theor. Phys. 122, 859 (2016).

[33] N.N. Bogolyubov, Dynamical Problems in Statistical Physics, Gostekhizdat, Moscow 1946 (in Russian).

[34] Y. Waseda, The Structure of Non-Crystalline Materials: Liquids and Amorphous Solids, McGraw-Hill, New York 1980.

[35] R.M. Khusntdinoff, A.V. Mokshin, B.A. Klumov, R.E. Ryltsev, N.M. Chtchelkatchev, J. Exp. Theor. Phys. 123, 265 (2016).

[36] A.V. Mokshin, R.M. Khusnutdinoff, A.G. Novikov, N.M. Blagoveshchenskii, A.V. Puchkov, JETP 121, 828 (2015).

[37] T. Scopigno, U. Balucani, G. Ruocco, F. Sette, J. Phys. Condens. Matter 12, 8009 (2000).

[38] A.V. Mokshin, R.M. Khusnutdinov, A.R. Akhmerova, A.R. Musabirova, JETP Lett. 106, 366 (2017). 